\title{
Electronic properties of Li-doped zigzag graphene nanoribbons
}

\author{
P. Narin ${ }^{\text {a,* }}$, E. Kutlu ${ }^{\text {a }}$, B. Sarikavak-Lisesivdin ${ }^{\text {a }}$, S.B. Lisesivdin ${ }^{\text {a }}$, E. Özbay ${ }^{\text {b,c,d }}$ \\ a Department of Physics, Faculty of Science, Gazi University, Teknikokullar, 06500 Ankara, Turkey \\ ${ }^{\mathrm{b}}$ Nanotechnology Research Center, Bilkent University, Bilkent, 06800 Ankara, Turkey \\ ${ }^{\mathrm{c}}$ Department of Physics, Bilkent University, Bilkent, 06800 Ankara, Turkey \\ d Department of Electrical and Electronics Engineering, Bilkent University, Bilkent, 06800 Ankara, Turkey
}

\section{H I G H L I G H T S}

- Electronic properties of Li-doped ZGNR are investigated.

- Spin polarized calculations are carried out with DFT.

- ZGNR is exhibited half metallic or semiconductor behavior.

\section{A R T I C L E I N F O}

\section{Article history:}

Received 8 December 2015

Received in revised form

30 May 2016

Accepted 20 June 2016

Available online 20 June 2016

Keywords:

Nanoribbon

Doping

Electronic properties

DFT

\begin{abstract}
A B S T R A C T
Zigzag graphene nanoribbons (ZGNRs) are known to exhibit metallic behavior. Depending on structural properties such as edge status, doping and width of nanoribbons, the electronic properties of these structures may vary. In this study, changes in electronic properties of crystal by doping Lithium (Li) atom to ZGNR structure are analyzed. In spin polarized calculations are made using Density Functional Theory (DFT) with generalized gradient approximation (GGA) as exchange correlation. As a result of calculations, it has been determined that Li atom affects electronic properties of ZGNR structure significantly. It is observed that ZGNR structure exhibiting metallic behavior in pure state shows half-metal and semiconductor behavior with $\mathrm{Li}$ atom.
\end{abstract}

(c) 2016 Elsevier B.V. All rights reserved.

\section{Introduction}

Since the discovery of graphene, intensive works have been carried out on this material [1-4]. Carbon nano-materials have become the focus of attention for the last 20 years because low dimensional characteristics and unique electronic properties [5,6]. Graphene nanoribbons (GNRs) exhibit quite significant features indicating that they will have high usage possibility for carbonbased nano-electronics [7-10]. GNRs have a one-dimensional structure. Their structural and electronic characteristics have been examined in detail in both experimental and theoretical studies [11]. GNR structures are of two types including Zigzag edged (ZGNR) and Armchair (AGNR) edged types [12]. AGNR structures are exhibiting metallic or semiconducting behaviors for their different widths [13-15]. ZGNRs are mostly behaving metallic. However, ZGNRs show edge-related magnetism [7,15,16]. Therefore, behavior of the band structure is much more complex than the AGNRs. According to some theoretical works, an applied

\footnotetext{
* Corresponding author.

E-mail address: polatnarin0@gmail.com (P. Narin).
}

electric field perpendicular to the nanoribbon itself, may change the behavior of the ribbon between a semiconducting and a halfmetallic state due to effect of the electric field on an antiferromagnetic edge state $[9,17]$.

GNR structures show opposite behavior exhibit metallic behavior compared to carbon nanotubes in terms of their electronic properties [18-20]. However, some theoretical studies may show different electronic properties of carbon nanotubes [14,20,21].

Electronic properties of GNR structures can be explained with both their edge states and nanoribbon width and are known to change with doping [22-25]. Because of having spintronic features, electronic properties of GNR structures can be analyzed based on spin [26,27]. Apart from metallic and semiconducting states, electronic band structure depending on different spin states, it is known that there are concepts such as half-metal and spin-gapless semiconductor [28]. Generally, the structure with one spin state with metallic behavior and other spin state with semiconductor behavior is called half metallic. If parallel spin or opposite spin bands touch each other at the Fermi point, this is called the spin-gapless semiconductor state [28]. And, half metal cases is usually observed in zigzag edged GNRs. Three properties can bring ZGNR structure to half metal state; changing of edges of ZGNR, 
doping with atoms or applying of an external electric field $[7,23,29-31]$.

Li atom substantially is used making energy storage and conversion such as lithium ion batteries (LIBs), supercapacitors and fuel cells in graphene-based nanomaterials [32,33]. Besides energy storage and conversion, LIBs have become important for movable electronic devices because of their rechargeable properties in last two decades [32]. Therefore other than graphene, using Li atoms with other carbon-based materials may affect the electronic properties of those materials significantly. Previously, Li atom was used as adatom in zigzag graphene nanoribbon [34-38]. To our knowledge, the electronic properties of substitutional Li doped GNRs were not studied yet.

In this study, the effect of doping of $\mathrm{Li}$ atom on electronic properties of ZGNR structures were analyzed theoretically with the help of motivation given by the possible important applications of Li interacted graphene-based nanomaterials.

\section{Calculation method}

Calculations were carried out using Atomistix Toolkit-Visual NanoLab (ATK-VNL) software based on the DFT calculations $[39,40]$. In spin polarized calculations have been carried out with generalized gradient approximation (GGA) and Perdew-BurkeErnzerhof (PBE) was used as exchange correlation functional [41]. In calculations, nano-ribbons terminated with hydrogen atoms with width $\left(N_{z}\right)$ between 4 and 12 has been used. However, since the results of the structures with different $N_{z}$ gave similar results, only results from the state where $N_{z}=8$ have been evaluated throughout the article. In calculations for the studied structures; cut-off energy and maximum force applied to the crystal are taken respectively as $300 \mathrm{eV}$ and $0.05 \mathrm{eV} / \AA$. In addition, the periodic boundary condition was accepted along $z$-axis and MonkhorstPack $1 \times 1 \times 100 \mathrm{k}$-point grid parameters have been used.

$E_{B}=E_{T}-E_{T: \text { bare }}-\mathrm{n} E_{H}-m E_{L i}$

$E_{F}=\left(E_{T: d o p}+E_{c}\right)-\left(E_{T: H}+m E_{L i}\right)$

Solving Eq. (1) and Eq. (2), it has been determined on which doping sites at 8-ZGNR structure is steady [49]. In Eq. (1), $E_{B}$ is binding energy, $E_{T}$ is the doped structure's total energy, $E_{T \text { :bare }}$ is total energy of ZGNR without finishing with any atoms, $E_{H}$ and $E_{L i}$ are free energy of a half of a isolated $\mathrm{H}_{2}$ molecule and a lithium atom, $n$ and $m$ are the number of $\mathrm{H}$ atoms and $\mathrm{Li}$ atom in the structure. In Eq. (2), $E_{F}$ is the formation energy, $E_{T: \text { dop }}$ is total energy of doped structure, $E_{c}$ is free energy of a carbon atom, $E_{T: H}$ is the total energy of the structure terminated with hydrogen.

\section{Results and discussion}

8-ZGNR structure used in calculations is represented in Fig. 1. In the cell, positions of possible Li doping sites are assigned with a letter from A to G, and for each site, electronic band structure and density of states (DOS) have been examined. As a result of Eqs. (1) and (2), formation energy and binding energy values given with in Fig. 2 have been determined. While low binding and low formation energies are observed in Li doping sites with even numbered lines as shown in Fig. 2, high binding and formation energies are observed in odd numbered lines.

In literature, it is known that Li atom to hollow site of graphene is more stable than that of bridge and top sites [43,44]. Binding and formation energies of $\mathrm{Li}$ atom doped to hollow site of graphene have been shown in Table 1. As shown, binding and

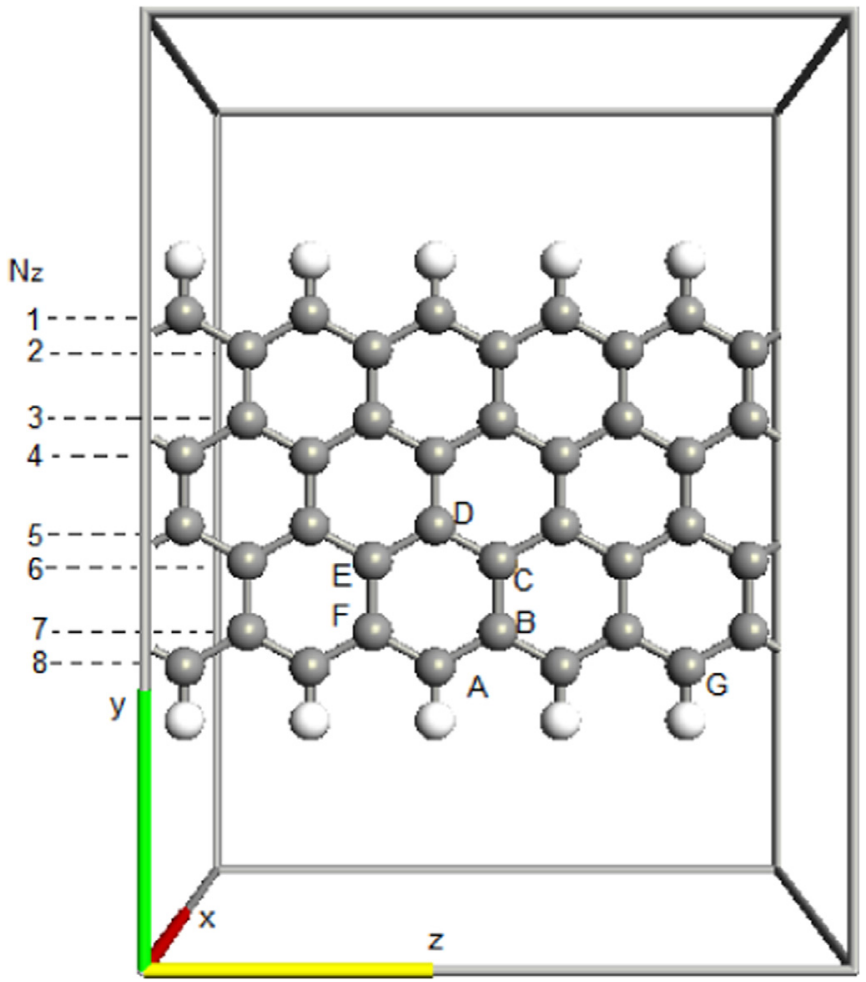

Fig. 1. Schematic view of Li dosing in different positions of nanoribbon, naming from letter $A$ to $G$ corresponds to doping positions in 8-ZGNR.

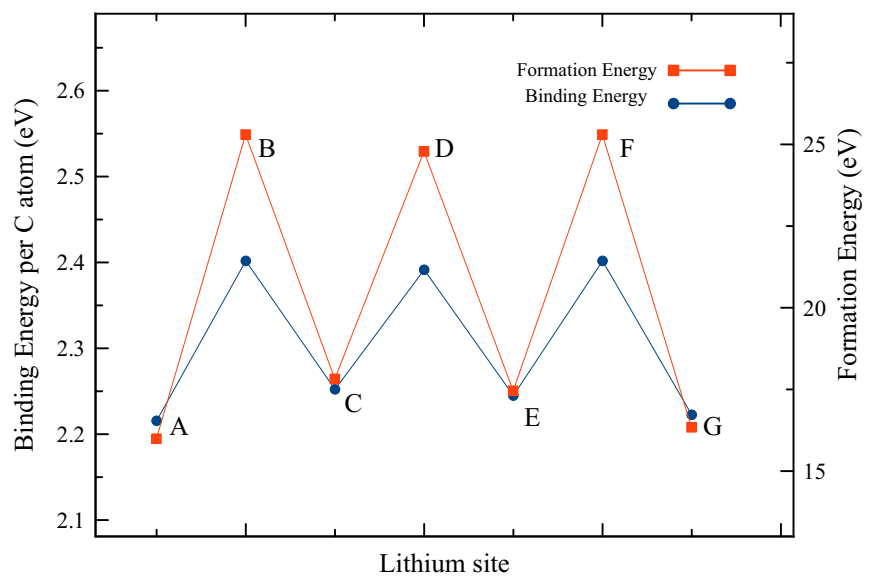

Fig. 2. Binding and formation energy changes depending on the position of Li atom within 8-ZGNR.

Table 1

For Li-doped structures, binding and formation energies of this and previously studies.

\begin{tabular}{lll}
\hline & Binding energy $(\mathrm{eV})$ & Formation energy $(\mathrm{eV})$ \\
\hline Graphene (hollow site) & & \\
& $1.60[42]$ & $7.83[46]$ \\
& $1.55[43]$ & $7.2-7.9[47]$ \\
This study & $1.93[45]$ & 15.99 \\
\hline
\end{tabular}

formation energies of Li doped graphene are lower than our results. However, binding energy of this study is stronger than that of graphene. This binding energy is consistent with previous study which is binding energy of Li adsorption on ZGNR [48].

In Fig. 3, electronic band structure and DOS of 8-ZGNR 
structure terminated with $\mathrm{H}$ atom are shown. For all electronic band structures in this study, spin up states are shown with continuous lines and spin down states are shown with dashed lines. The pure structure terminated with $\mathrm{H}$ atoms exhibits metallic behavior. From DOS, it is understood that ZGNR structures have spintronic properties.
Electronic band structures for each spins where Li impurity atom is placed to sites from $A$ to $D$ are given in Fig. 4. In electronic band structure, $\mathrm{Li}$ atom at A site can be said to behave as metallic for spin up bands, and as n-type semiconductor for spin down bands. Considering the $\mathrm{Li}$ atom at $\mathrm{B}$ site, it is apparent that it has more half-metal band structure and it is metallic for spin up bands

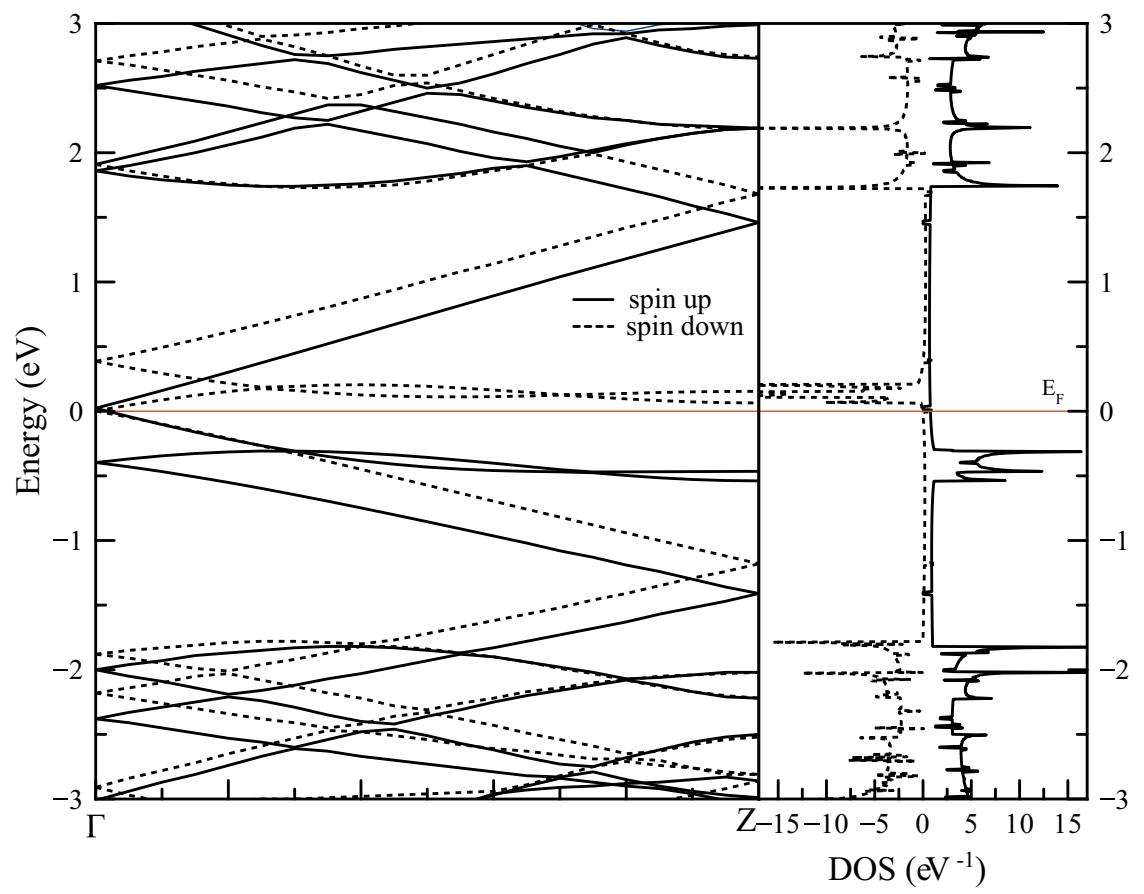

Fig. 3. Electronic band structure and DOS of 8-ZGNR structure terminated with $\mathrm{H}$ atom.

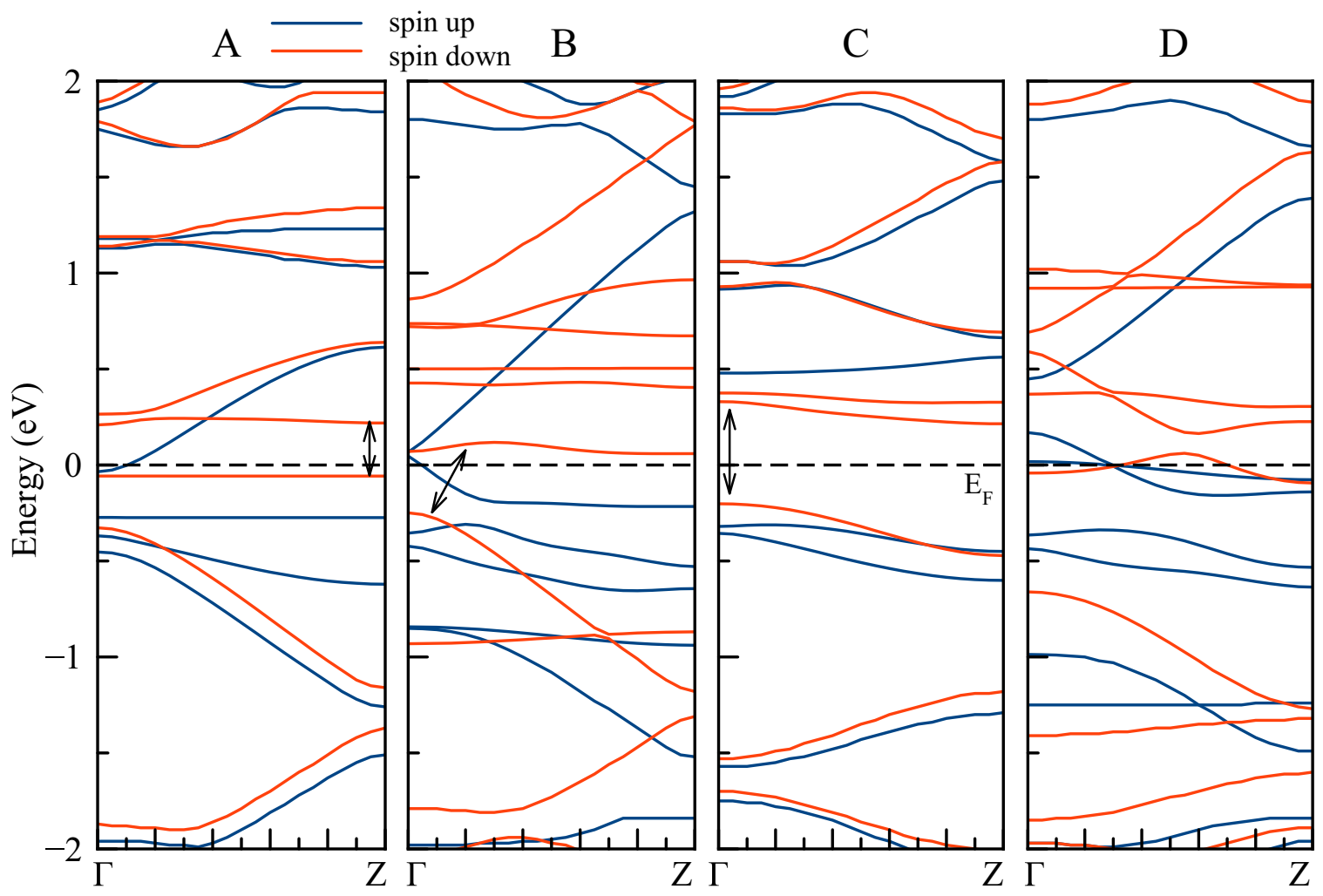

Fig. 4. Band structures of $8-Z G N R$ structures for $A, B, C, D$ configurations. 
and semiconductor for spin down bands. Considering the band structure for $\mathrm{C}$ site, a semiconductor behavior with having direct forbidden gap in spin up bands and indirect forbidden gap in spin down bands is observed. Forbidden band gaps for spin up and spin down bands are determined as $0.79 \mathrm{eV}$ and $0.41 \mathrm{eV}$, respectively. It is observed that the band structure of Li impurity atom for D site behaves as metallic for both spin up and spin down bands as in spin up bands of A site. As shown in Fig. 1, A, B and C sites are structurally symmetrical with the G, F and E, respectively. Electronic behaviors as band structure and DOS of G, E, F states have observed the same electronic properties with A, B and C. Therefore bant structures and DOS of these states have not been shown.

In Fig. 5(a) and (b), total DOS and PDOS of Li impurity atom at A site are given, respectively. Considering PDOS chart of Li impurity atom at A site with half-metallic behavior, there is no any density near Fermi level. Therefore, Li impurity atom does not contribute to the conduction. In Fig. 6(a) and (b), total DOS and PDOS of Li impurity atom at B site are given, respectively. No density is observed around Fermi level for PDOS of Li impurity atom.

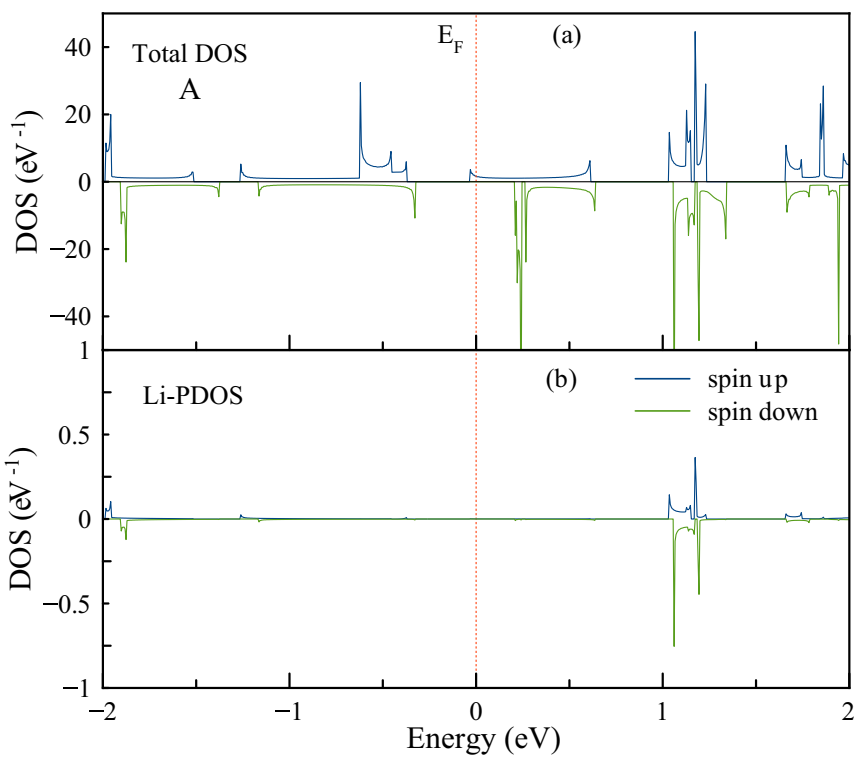

Fig. 5. (a, b) Total DOS and PDOS of Li atom in A site.

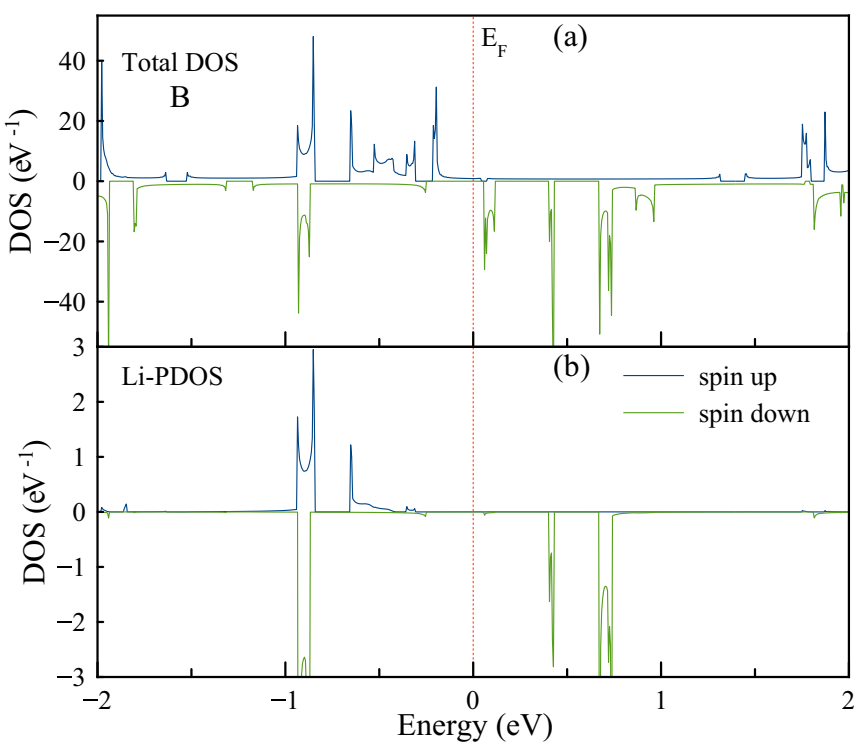

Fig. 6. (a, b) Total DOS and PDOS of Li atom in B site.

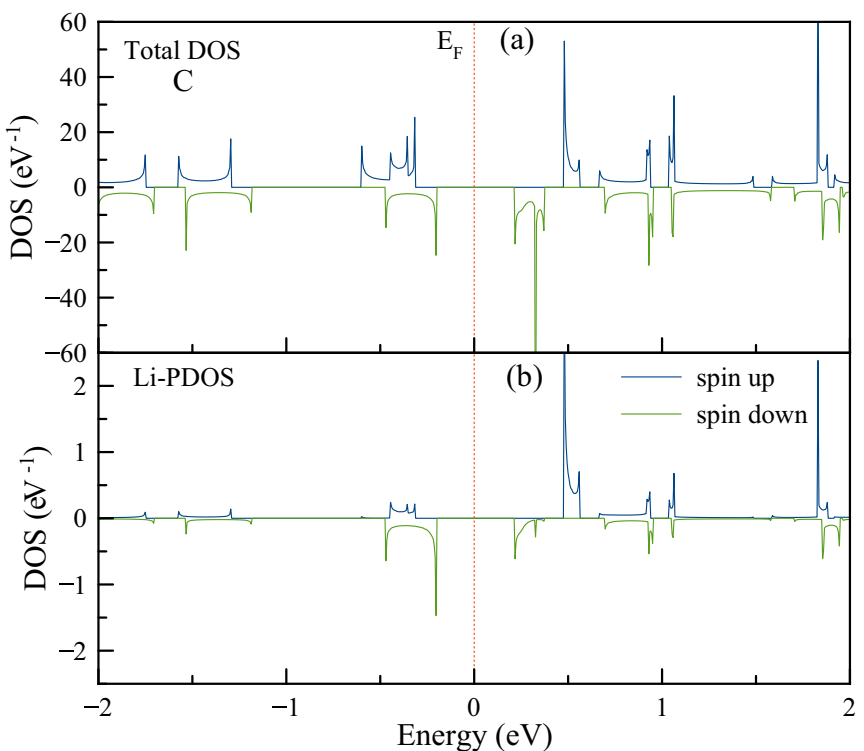

Fig. 7. (a, b) Total DOS and PDOS of Li atom in C site.

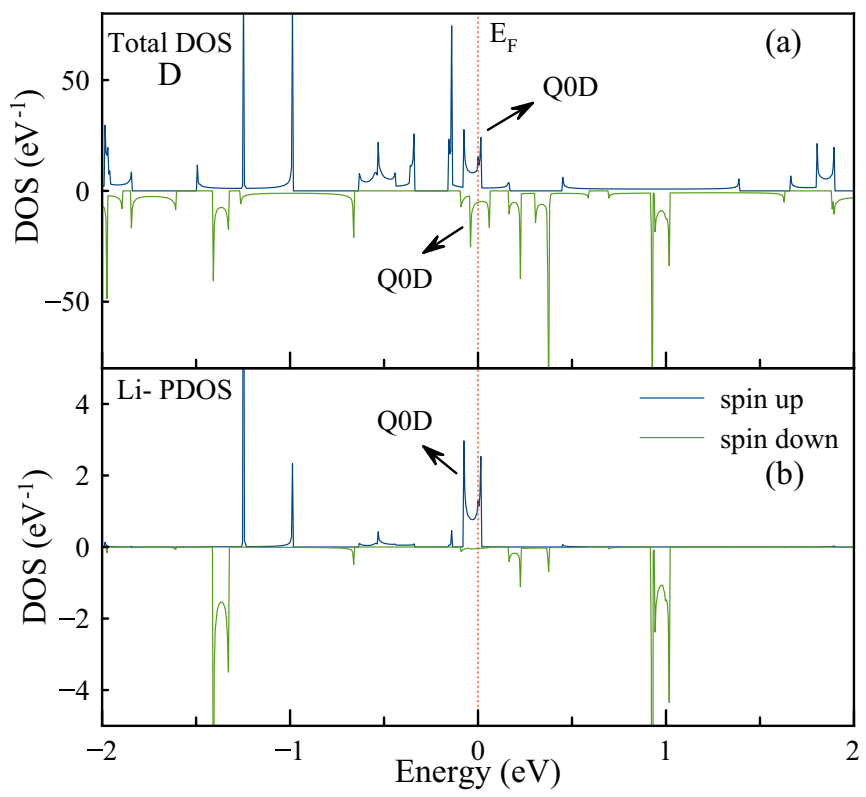

Fig. 8. (a, b) Total DOS and PDOS of Li atom in D site.

In Fig. 7(a) and (b), total DOS and PDOS of Li impurity atom at C site are given, respectively. It is observed that the structure exhibits semiconductor behavior and the valance band maximum and conduction band minimum is found for spin down case. Therefore, the conduction of system is spin-selected. Same behavior is also observed at PDOS of Li impurity atom. In Fig. 8(a) and (b), total DOS and PDOS of Li impurity atom at D site are given, respectively. According to Fig. 8(b), the structure exhibits metallic behavior and this behavior caused by spin-up state contribution of Li impurity atom. In Fig. 8, Quasi-zero-dimensional (Q0D) behavior has been shown at Fermi point. Q0D are merged of 1D and OD behaviors $[49,50]$.

\section{Conclusion}

Effects of Li impurity atom doped within zigzag edged graphene nanoribbon structure on the electronic properties of ZGNR 
structure have been analyzed with DFT method based on spin type. 8-ZGNR structure with calculations shown is terminated with $\mathrm{H}$ atoms and it has exhibited metallic behavior in undoped state. Depending on positions of the impurity atom, metallic, halfmetal or semiconductor behaviors are observed. When Li atom is at $\mathrm{A}$ site, it is determined to be in the most steady state. In addition, the partial densities of state (PDOS) has been analyzed with a view to determining contributions from Li impurity atom and to determine total state density for each state. It has been observed that Li impurity atom changes electronic properties of ZGNR considerably. Especially, because of observed spin-selected semiconductor behavior for the Li impurity located at $\mathrm{C}$ site, the structure may have important applications in spintronics research. Also the Li impurity atom can be help controlling electronic features of the ZGNR as a semiconductor or a half metal.

\section{Acknowledgments}

This work is supported by the projects DPT-HAMIT, DPT-FOTON, and NATO-SET-193 and TUBITAK under the project Nos. 113E331, 109A015 and 109E301. One of the authors (E.O.) also acknowledges partial support from the Turkish Academy of Sciences.

\section{References}

[1] K.S. Novoselov, A.K. Geim, S.V. Morozov, D. Jiang, Y. Zhang, S.V. Dubonos, I V. Grigorieva, A.A. Firsov, Science 306 (2004) 666.

[2] Y. Zhang, Y.W. Tan, H.L. Stormer, P. Kim, Nature 438 (2005) 201.

[3] K.S. Novoselov, A.K. Geim, S.V. Morozov, D. Jiang, M.I. Katsnelson, I. V. Grigorieva, S.V. Dubonos, A.A. Firsov, Nature 438 (2005) 197.

[4] K.S. Novoselov, Z. Jiang, Y. Zhang, S.V. Morozov, H.L. Stormer, U. Zeitler, J. C. Maan, G.S. Boebinger, P. Kim, A.K. Geim, Science 315 (2007) 1379.

[5] M.S. Dresselhaus, G. Dresselhaus, P.C. Eklund, Academic press, 1996.

[6] L. Chico, V.H. Crespi, L.X. Benedict, S.G. Louie, M.L. Cohen, Phys. Rev. Lett. 76 (1996) 971.

[7] Y.W. Son, M.L. Cohen, S.G. Louie, Nature 444 (2006) 347.

[8] K. Wakabayashi, Phys. Rev. B 64 (2001) 125428.

[9] V. Barone, O. Hod, G.E. Scuseria, Nano Lett. 6 (2006) 2748.

[10] D.A. Areshkin, D. Gunlycke, C.T. White, Nano Lett. 7 (2007) 204.
[11] X. Li, X. Wang, L. Zhang, S. Lee, H. Dai, Science 319 (2008) 1229.

[12] L. Yang, C.H. Park, Y.W. Son, M.L. Cohen, Steven G. Louie, Phys. Rev. Lett. 99 (2007) 186801

[13] M. Ezawa, Phys. Rev. B 73 (2006) 045432.

[14] K. Wakabayashi, M. Fujita, H. Ajiki, M. Sigrist, Phys. Rev. B 59 (1999) 8271.

[15] M. Fujita, K. Wakabayashi, K. Nakada, K. Kusakabe, J. Phys. Soc. Jpn. 65 (1920) (1996).

[16] O. Hod, V. Barone, G.E. Scuseria, Phys. Rev. B 77 (2008) 035411.

[17] K.N. Kudin, ACS Nano 2 (2008) 516.

[18] K. Nakada, M. Fujita, G. Dresselhaus, M.S. Dresselhaus, Phys. Rev. B 54 (1996) 954.

[19] V. Barone, O. Hod, G.E. Scusera, Nano Lett. 6 (2006) 2748.

[20] Y.L. Lee, Y.W. Lee, Phys. Rev. B 66 (2002) 245402.

[21] Y. Miyamoto, K. Nakada, M. Fujita, Phys. Rev. B 59 (1999) 9858.

[22] S.S. Yu, W.T. Zheng, Q.B. Wen, Q. Jiang, Carbon 46 (2008) 537.

[23] Y. Li, Z. Zhou, P. Shen, Z. Chen, ACS Nano 3 (2009) 1952.

[24] N.K. Jaiswal, P. Srivastava, Physics E 44 (2011) 75.

[25] N.K. Jaiswal, P. Srivastava, Solid State Commun. 152 (2012) 1489.

[26] W.Y. Kim, Y.C. Choi, K.S. Kim, J. Mater. Chem. 18 (2008) 4510.

[27] D. Soriano, F. Muñoz-Rojas, J. Fernández-Rossier, J.J. Palacios, Phys. Rev. B 81 (2010) 165409.

[28] X.L. Wang, Phys. Rev. Lett. 100 (2008) 156404.

[29] Z. Li, J. Yang, J.G. Hou, J. Am. Chem. Soc. 130 (2008) 4224

[30] X.H. Zheng, X.L. Wang, T.A. Abtew, Z. Zeng, J. Phys. Chem. C 114 (2010) 4190.

[31] E.J. Kan, Z. Li, J. Yang, J.G. Hou, Appl. Phys. Lett. 91 (2007) 243116.

[32] N. Mahmood, C. Zhang, H. Yin, Y. Hou, J. Mater. Chem. A 2 (2014).

[33] Y. Jing, Z. Zhou, C.R. Cabrera, Z. Chen, J. Mater. Chem. A 2 (2014) 12104.

[34] C. Uthaisar, V. Barone, J.E. Peralta, J. Appl. Phys. 106 (2009) 113715.

[35] C. Uthaisar, V. Barone, Nano Lett. 10 (2010) 2838.

[36] A.M. Garay-Tapia, A.H. Romero, V. Barone, J. Chem. Theory Comput. 8 (2012) 1064.

[37] X. Fan, W.T. Zheng, J.L. Kuo, ACS Appl. Mater. Interfaces 4 (2012) 2432.

[38] D. Krepel, O. Hod, J. Phys. Chem. C 117 (2013) 19477.

[39] Version 12.2.2 QuantumWise A/S. 〈http://www.quantumwise.com〉.

[40] J.M. Soler, E. Artacho, J.D. Gale, A. Garcia, J. Junquera, P. Ordejon, D. SanchezPortal, J. Phys.: Condens. Matter 14 (2002) 2745.

[41] K. Burke, J.P. Perdew, M. Ernzerhof, Int. J. Quantum Chem. 61 (1997) 287.

[42] M. Khantha, N.A. Cordero, L.M. Molina, J.A. Alonso, L.A. Girifalco, Phys. Rev. B 70 (2004) 125422.

[43] F. Valencia, A.H. Romero, F. Ancilotto, P.L. Silvestrelli, J. Phys. Chem. B 110 (2006) 14832.

[44] K.T. Chan, J.B. Neaton, M.L. Cohen, Phys. Rev. B 77 (2008) 235430.

[45] C. Ataca, E. Aktürk, S. Ciraci, H. Ustunel, Appl. Phys. Lett. 93 (2008) 043123.

[46] L.J. Zhou, Z.F. Hou, L.M. Wu, J. Phys. Chem. C 116 (2012) 21780.

[47] H. Yildirim, A. Kinaci, Z.J. Zhao, M.K.Y. Chan, J.P. Greeley, ACS Appl. Mater Interfaces 6 (2014) 21141.

[48] C. Uthaisar, V. Barone, J.E. Peralta, J. Appl. Phys. 106 (2009) 113715.

[49] B. Sarikavak-Lisesivdin, S.B. Lisesivdin, E. Ozbay, Mol. Phys. 110 (2012) 2295.

[50] A.F. Kuloglu, B. Sarikavak-Lisesivdin, S.B. Lisesivdin, E. Ozbay, Comput. Mater. Sci. 68 (2013) 18 